\title{
Transcription factor-induced epigenetic reprogramming
}

\author{
Konrad Hochedlinger ${ }^{1}$ \\ ${ }^{I}$ Massachusetts General Hospital Cancer Center and Center for Regenerative Medicine, Harvard Stem Cell Institute, Boston, \\ Massachusetts, 02114, USA
}

\begin{abstract}
Mammalian development has been thought to be a one-way process, which starts with a few embryonic founder cells, that become more and more restricted and ultimately give rise to all specialized cell types of the body. The cloning of the sheep Dolly from an adult mammary gland cell has refuted this dogma and demonstrated that the developmental clock of a mature cell can be "reprogrammed" by the egg into that of an embryonic cell, which can support development of a clone. Reprogramming research has enormous therapeutic potential in humans as it may allow for the derivation of embryonic stem (ES) cells from patients' cells suffering from degenerative disorders such as Alzheimer's disease, Parkinson's disease or diabetes; because ES cells have the ability to give rise to all cell types of the body when exposed to the right combination of growth factors, these cells may provide a unique source of replacement tissue for regenerative medicine. Because of the legal, ethical and logistic restraints of nuclear transfer, it would be desirable to find alternative ways to generate patient-specific stem cells. Indeed, a recent discovery indicated that ectopic expression of the four transcription factors Oct4, Sox2, c-Myc, and Klf4 is sufficient to confer a pluripotent state upon the fibroblast genome, generating induced pluripotent stem (iPS) cells. However, these cells were not identical to ES cells produced from fertilized embryos in their transcriptional and epigenetic states and their developmental potential. Using novel selection approaches, we have recently generated iPS cells directly from fibroblasts and characterized their epigenetic state and differentiation potential. Like ES cells, female iPS cells showed reactivation of a somatically silenced $X$ chromosome and underwent random $X$ inactivation upon differentiation. Moreover, genome-wide analysis of two key histone modifications, H3K4 and H3K27 trimethylation, indicated that iPS cells are highly similar to ES cells. Consistent with these observations, iPS cells gave rise to viable high degree chimeras with contribution to the female germ line. Together, these data show that transcription factorinduced reprogramming leads to the global reversion of the somatic cell epigenome into an ES-like state.
\end{abstract}

Keywords: Epigenetic Reprogramming, iPS cells, Oct4, Sox2, c-myc, Klf4

Cell Research (2008) 18:s4. doi: 10.1038/cr.2008.94; published online 4 August 2008

Correspondence: Konrad Hochedlinger

E-mail: khochedlinger@helix.mgh.harvard.edu

Konrad Hochedlinger, PhD, is an Assistant Professor of Medicine at Harvard Medical School, a Principal Faculty Member at the Harvard Stem Cell Institute and a Member of the Massachusetts General Hospital Cancer
Center and Center for Regenerative Medicine. He received his undergraduate training at the University of Vienna/Austria and his $\mathrm{PhD}$ and Postdoctoral education at MIT/Whitehead Institute. He is a Kimmel Scholar and a V Scholar and the recipient of the Inaugural NIH Director's Innovator Award. Research in Dr. Hochedlinger's lab is aimed at understanding the molecular basis of epigenetic reprogramming and pluripotency. 